\title{
Midface Reconstruction: Planning and Outcome
}

\author{
Gautam Biswas $^{1}$ \\ ${ }^{1}$ Department of Plastic and Reconstructive Surgery, TATA Medical \\ Center, Kolkata, West Bengal, India
}

\begin{abstract}
Address for correspondence Gautam Biswas, MS, MCh, DNB, Department of Plastic and Reconstructive Surgery, TATA Medical Center, Kolkata, West Bengal 700156, India (e-mail: dr.biswas@gmail.com).
\end{abstract}

\begin{abstract}
Keywords

- midface

- maxilla reconstruction

-3D planning

- nasal reconstruction

Reconstruction of the complex anatomy and aesthetics of the midface is often a challenge. A careful understanding of this three-dimensional (3D) structure is necessary. Anticipating the extent of excision and its planning following oncological resections is critical.

In the past over two decades, with the advances in microsurgical procedures, contributions toward the reconstruction of this area have generated interest. Planning using digital imaging, 3D printed models, osseointegrated implants, and low-profile plates, has favorably impacted the outcome. However, there are still controversies in the management: to use single composite tissues versus multiple tissues; implants versus autografts; vascularized versus nonvascularized bone; prosthesis versus reconstruction.

This article explores the present available options in maxillary reconstruction and outlines the approach in the management garnered from past publications and experiences.
\end{abstract}

\section{Introduction}

The midface is the anatomical region of the face, with the maxilla as its bony support and in close relation with the skull base, oral cavity, orbit, and the nose. By virtue of its unique location, it is both anatomically complex and functionally crucial. Its structural integrity is vital to normal breathing, speech, swallowing, orbital support, and alignment of the globe, and contributes to the facial aesthetics.

Oncologic defects involving this area are complex, requiring a three-dimensional (3D) approach to reconstruction, and most options available can at best satisfy only some of the reconstructive goals.

The aim of this article is to present an overview of understanding the reconstructive options in such defects and the $3 \mathrm{D}$ planning required. The outcomes as well as some major limitations of using currently available techniques are also analyzed.

\section{Aims of Midface Reconstruction}

With a better understanding of tumor pathology and the availability of effective adjuvant treatment, resections in this complex area have also become extensive and with concomitant progress in reconstructive techniques, particularly microsurgical procedures, ${ }^{1}$ most if not all such defects can be reconstructed.

The major aim of most reconstructive options is to:

- restore the facial features.

- segregate the skull base from the nasal and oral cavity.

- restore upper jaw dentition and

- provide orbital support, if required.

The literature is replete with the successful use of various innovative techniques utilizing vascularized and nonvascularized bone, soft tissue, implants such as titanium mesh and porous polyethylene, in addressing such reconstructions. ${ }^{1-5}$ published online

December 31, 2020
DOI https://doi.org/

10.1055/s-0040-1721870

ISSN 0970-0358.
C2020. Association of Plastic Surgeons of India.

This is an open access article published by Thieme under the terms of the Creative Commons Attribution-NonDerivative-NonCommercial-License, permitting copying and reproduction so long as the original work is given appropriate credit. Contents may not be used for commercial purposes, or adapted, remixed, transformed or built upon. (https://creativecommons.org/licenses/by-nc-nd/4.0/). Thieme Medical and Scientific Publishers Pvt. Ltd. A-12, 2nd Floor, Sector 2, Noida-201301 UP, India 
In comparison with mandibular reconstructions, midface resections or the various types of maxillectomies are less common, and considering the spectrum of defects and varied approaches and options for management, there is no clear cut consensus for the best option in most defects. ${ }^{6}$ Most reconstructions aim at restoring the facial features, at segregating the cranial from the nasal and oral cavity, restoring upper jaw dentition, and providing orbital support where required.

Achieving an optimal result in a single stage is often challenging in mid-face reconstructions. Obtaining such a result using a single flap, comprising multiple components of skin islands, muscles, and multiple segments of bone makes the exercise more daunting.

Key principles in midfacial reconstruction are:

- Creating the upper bony alveolus.

- Creating its buttresses wherever possible.

- Minimizing the dead space.

- Providing an epithelial palatal barrier.

- Providing an epithelial cover (external) wherever this is deficient.

- Orbital floor support.

Dealing with the orbital floor defect where the globe itself is not involved is one area where there are different approaches, one of the commonest being use of the vascularized fibula for the orbital rim, with or without extensions to replace the floor. These extensions may be implants like titanium mesh or neovascularized bone. ${ }^{6}$

\section{Maxillary Reconstruction}

The maxillae constitute the main part of the midfacial bony structure.

Attempts at organizing and categorizing the various defects following mid facial resections, with suggestions for possible reconstructive options have led to the formulation of several classification systems. ${ }^{7-12}$ There are key differences and limitations in each of these classifications. For example, Cordeiro's classification does not address central defects. ${ }^{1}$ For complex defects Cordeiro's types IIIa, IIIb, and IV, soft tissue free flaps are suggested, such as the rectus abdomens free flap while Brown proposed deep circumflex iliac artery (DCIA) flap, emphasizing that bony support is needed to prevent a gravity-induced shift of the soft tissue..$^{8,9}$ The proposed reconstructive algorithm also does not address patients with nasal defects and those who need dental rehabilitation. ${ }^{6}$

Schematically the maxilla may be visualized as a cuboidal structure with its walls enclosing a space lined with epithelium. The floor constitutes the palatal shelf with its epithelized oral and nasal surfaces. A minimum reconstructive goal is to replace this with a bony alveolar ridge and a vascularized skin flap to bridge the oral cavity roof defect. Brown proposed placing the DCIA flap horizontally in a near-total palatal defect (Class 2b-c defects). Palatal shelf reconstruction using bone is not necessary, and the alveolar ridge suffices both as a buttress force transmitter as well as providing adequate bony stock for osteointegrated implants. The limitations are that the nasal lining is left raw allowing it to heal by secondary intent, and the loss of the dynamic portion of the soft palate is not addressed. For small palatal defects, this is adequate, but speech and swallowing are often altered and delayed if a significant portion of the palate is missing.

In general, the practice often followed is to replace small defects (Brown type I or II) with soft tissue flaps, either free or pedicled or to use obturators.

\section{Obturators}

Prostheses have been in use for decades to create internal support as well as obturation and separation of the nasal and oral cavities. Though simple in concept, it requires a somewhat complex design especially if a major part of the maxilla is lost. Designs may involve multiple segments interlocked with each other. This is done as introducing some of these obturators through the oral aperture,may be difficult. Accurate fitment is also an issue, with frequent complaints of leakages, regurgitation, excoriation, and problems in the maintenance of oral hygiene. ${ }^{13}$

Studies comparing obturators and reconstruction have shown comparable results with respect to speech and swallowing between the two modalities. However, for larger defects, or when the anterior palate along with both the canines is removed, microsurgical reconstructions fare better. ${ }^{14,15}$

Inconvenience, feeling of discomfort, and self-consciousness with the use of prosthesis all possibly tilt the choice toward reconstructions. A prosthesis may be useful as a temporary measure if secondary reconstructions are planned or there is a waiting period due to unavoidable factors. In addition, prosthesis and obturators may also act as templates for the reconstructive surgeon to understand the volume and structure of the construct to be planned. ( Fig. 1A-C).

There is general agreement that for Cordeiro type I and II and Browns Class 1 or 2a defects the choice may range from an obturator, soft tissue, or vascularized bone such as the osteocutaneous fibula, radial forearm, or DCIA flaps. The choice rests on the individual surgeon's preference..$^{1,8,9,15}$ The "sandwich flap" incorporating vascularized bone between two layers of the radial forearm free flap (RAFF) described by Cordeiro and Santamaria is one such example which gives anterior facial projection, facial width and height, soft tissue support, and an option for dental rehabilitation. ${ }^{16,17}$ The consensus is less clear on the Cordeiro type III and IV defects and those involving the orbital floor ${ }^{16}$ where the choice of procedures ranges from soft tissue reconstructions, alone, either pedicled or free, osteocutaneous free flaps, combinations of soft tissue with nonvascularized bone or implants, or double free flaps. ${ }^{1,6}$

Large volume midfacial defects are best addressed using microsurgical procedures.

As more structural support is removed, bony replacement becomes essential. The osteocutaneous RAFF, scapula, fibula, $\operatorname{DCIA}(1,13,16,19,21)$ are some of the options. The cuboidal 

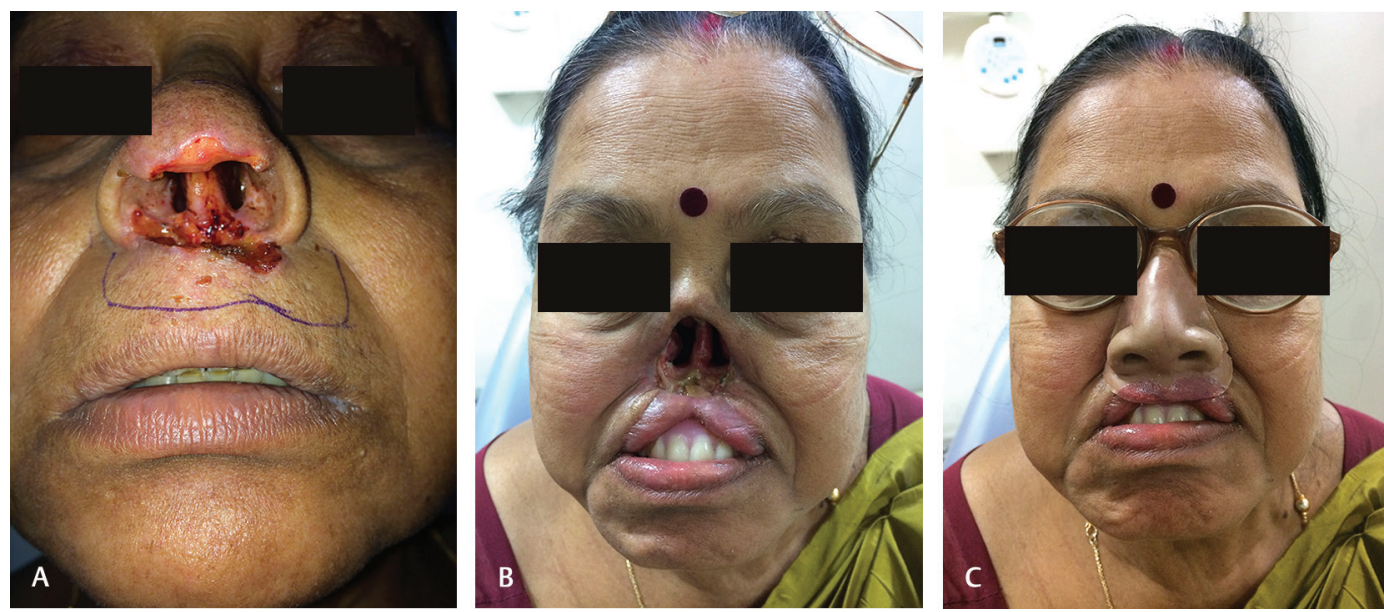

Fig. 1 (A) Squamous cell carcinoma nose and lip. (B) Post excision. (C) Replacement with a spectacle supported nasal and labial prosthesis. The patient was unwilling for further surgery.

bony structure is difficult to replicate and so bony support involves recreating horizontal and partial vertical buttresses.

The scapular system allows the transfer of multiple components, including:

- Skin islands based on transverse and descending branches from the circumflex scapular vessels.

- Soft tissue-latissimus dorsi and serratus muscle and

- Bone-lateral scapular margin.

The obvious limitation is in the positioning of the patient and inability to harvest the flap simultaneously with the resection team, in the supine position. Change of position would then need to follow the ablative procedure, which delays the procedure. ${ }^{17,18}$

Brown recommended the DCIA, which provided adequate bone for support of the cheek skin, a foundation for the creation of the orbital floor using nonvascularized bone or titanium implants, and also adequate bone stock for dental implants. ${ }^{19}$ He recommended placing the bone horizontally, creating a bony shelf, in infrastructure maxillary or palatal defects. When the defect is more vertical, the bone is positioned vertically with the crest forming the alveolar border. The internal oblique muscle harvested along with the flap fills the maxillary cavity and can also be used to fill the orbital cavity in case of orbital exenteration. The limitations are a short pedicle often requiring vein grafts, the internal oblique muscle which separates the nasal from the oral cavity requiring some time to epithelize, difficulty in designing multiple skin paddles to line the cavities created, and possible donor site problems. ${ }^{1,4,9,17}$

The fibular osteocutaneous flap has many proponents because of the ease of harvest, convenient two-team approach, longer pedicle, lending itself to multiple osteotomies, providing adequate bone for dental implants and carrying multiple soft tissue components, both skin and muscle, based on independent perforators ${ }^{4,9,20}$ (-Fig. 2). The difficulties arise in attempting to recreate the zygomatic complex, orbital rim, and orbital floor. ${ }^{4,20}$ In a study of 34 patients of maxillary reconstruction, of which

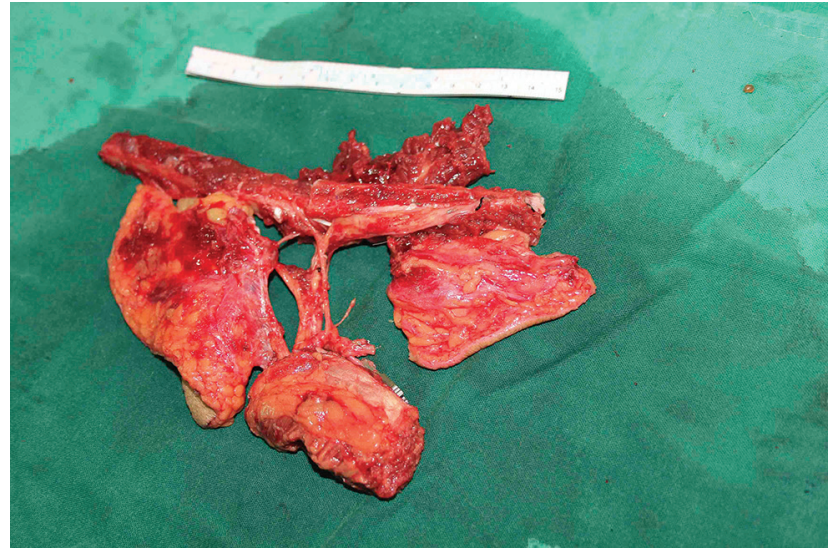

Fig. 2 Chimeric fibula osteocutaneous flap.

28 were Brown type I and II, the osteocutaneous fibula provided the maxillary form adequately, including the oronasal segregation.

In those with type III or type IV defects, this composite flap was "inadequate." The authors go on to suggest that in such situations, soft tissue flaps, such as the anterolateral thigh or rectus abdominis flap are preferred. ${ }^{4}$

Use of soft tissue alone results in the tissues sagging due to gravity leaving a void in the cheek where fullness is required. Some structural support is necessary to prevent this. ${ }^{21}$ The authors combine a free soft tissue and a second free vascularized bony flap which addresses the problems to some degree. Attempting to address the 3D composite defect can complicate the surgery. The plan is to reconstruct the horizontal and part of the vertical buttresses, provide a lining to separate the oral and nasal cavities, create the lateral nasal wall and also fill the potential dead space left following the maxillectomy. Chimeric flaps, based on either the peroneal vascular system or the thoracodorsal pedicle have been used. ${ }^{3}$

Chimeric flaps are governed by the length and lie of their vascular perforators. Trying to fit these small flaps, like a jigsaw puzzle, is not only challenging, but there are risks of kinking and twisting with loss of that component (-Fig. 2). 


\section{Double Flaps and Reconstructing the Inner Maxillary Defect}

Single composite tissue transfers, containing multiple component tissues though an option, is a challenge in planning and execution. The inner space created after maxillectomy is either left as it is when the focus is on creating the anterior maxillary shell, This may potentially mucosalize with time. However, this leaves a large raw area within, which can be obliterated by including muscle along with bone (soleus with fibula or internal oblique with iliac bone). The fate of these small muscle flaps is not clear as they would also sag and atrophy with time. A persistent nasal discharge is often the result of leaving a large raw area within. The vertical rectus abdominis, the anterolateral thigh with vastus lateralis has long vascular pedicles and can also carry independent skin and muscle islands. Muscle only flaps may be used for filling the inner cavity and creating a barrier between the nasal and oral cavity. The raw surfaces of these muscles on the maxillary floor and lateral wall mucosalize with time. ${ }^{6}$

It is reported that myocutaneous flaps bulge intraorally unless supported by bone in contrast to muscle only flaps which contract, mucosalize, and become concave. ${ }^{11}$ If supported the myocutaneous flaps also behave similarly, as the inner surface contracts. The authors combine two free flaps, the fibula to reconstruct the alveolar segment, the horizontal buttress, and its soft tissue to address the palatal defect. Combined with this, a second flap such as the rectus abdominis, or latissimus dorsi, can be used to fill the post maxillectomy cavity and its skin component as lining for the lateral nasal wall. The long pedicle from each of these flaps easily reaches the neck ${ }^{6}$ ( Fig. $\mathbf{3 A}, \mathbf{B}$ ).

The use of double flaps makes reconstruction of these complex 3D defects simpler. It is easier to inset these tissues, provide adequate volume, avoid kinks in its pedicle due to complicated positioning. Both flaps are elevated simultaneously as the head and neck team completes the excision and neck dissections. The fibula is also contoured and plated while in situ, ready for detachment and transfer. The author has found that this saves time and improves the efficiency of the team and allows manpower to be utilized optimally.

A premade gutter splint of the upper alveolus is often used as a template during in situ alveolus recreation ${ }^{22}$ (-Fig. 4).

In complex maxillectomy defects (Brown type III to IV), the fibula is widely used in practice for both the alveolus as well as the orbital margin. Using the fibula, which on an average provides approximately $22 \mathrm{~cm}$ length of bone, creation of the alveolar arch and placing a vascularized segment for the orbital margin, consume most of the available bone, reducing the vascular pedicle length to 8 to $9 \mathrm{~cm}$. The shortened vascular pedicle in such situations reaches just up to the lower mandibular border. Anastomosing at this point creates a gross vessel mismatch between the peroneal vessels and the facial vessels. Sometimes vein grafts may be necessary.

While using the fibular flap, the bone may be contoured to match the alveolar arch or placed obliquely for zygomatic maxillary buttress reconstruction. While creating the orbital rim the bones may be placed either in a $\mathrm{V}$ or a $\mathrm{U}$ pattern, removing a segment in between to allow for an easy lie of the vascular pedicle $6^{6,20}$ ( Fig. 5A-D).

Placed horizontally, it does address the horizontal buttress, but there is malalignment with regard to the lower jaw, with difficulties in dental rehabilitation. It also creates subtle soft tissue discrepancies with masking of the malar fullness. ${ }^{23}$ The authors feel that the alveolar arch needs to be aligned correctly and stabilized with the pterygoid plates if present, or by extending the transversally placed bone with a short vertical component fixed to the malar complex. The intervening area between the two horizontal constructs (orbital rim and alveolar ridge) which normally is a thin lamellar bridge also needs replacement as otherwise, the overlying soft tissue

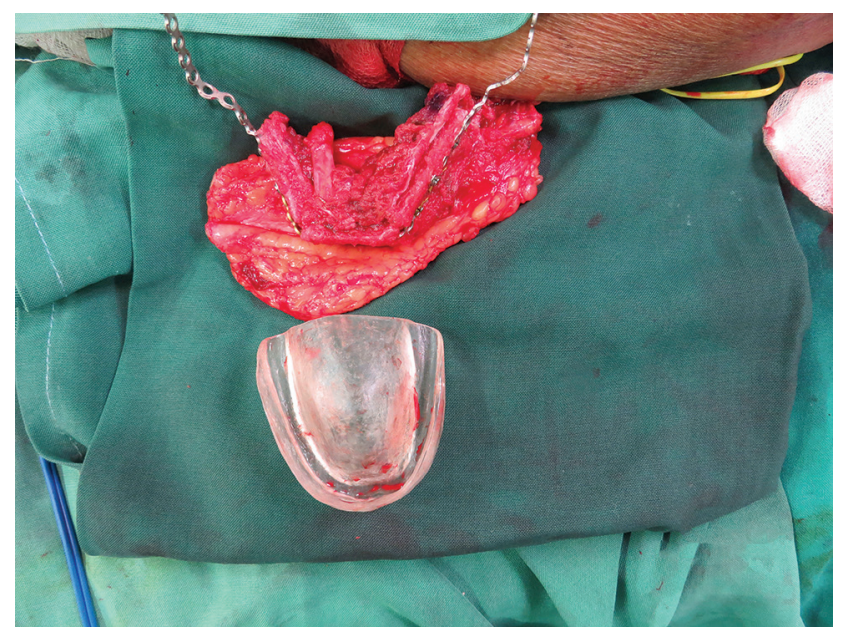

Fig. 4 Contouring of free fibula flap prior to pedicle detachment, using a premade dental gutter splint as reference.
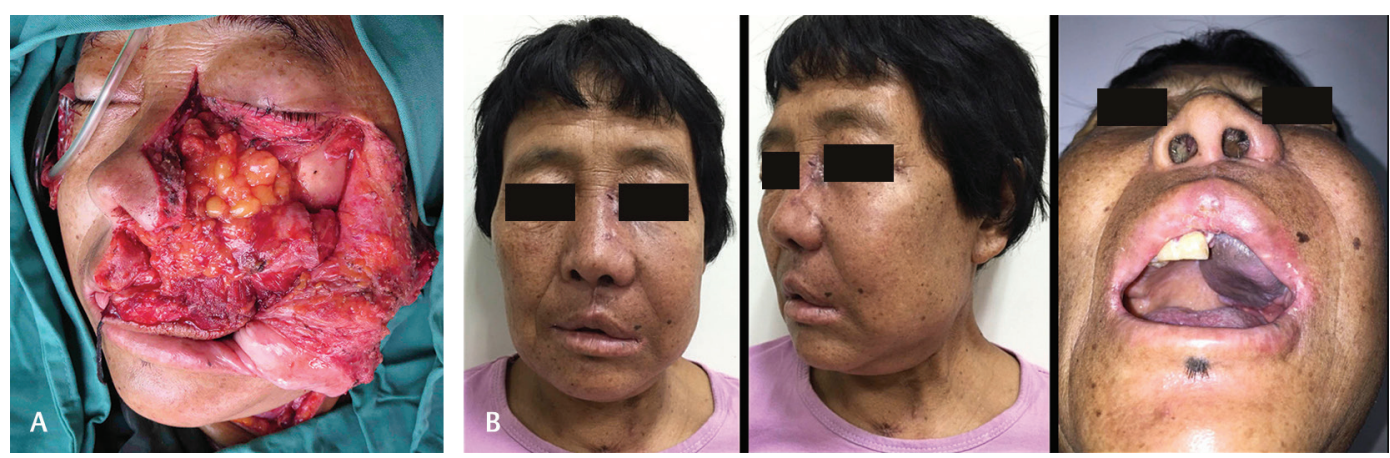

Fig. 3 (A, B) Double free flap reconstruction using a fibula osteocutaneous and a rectus abdominis flap for a type III maxillectomy defect. 

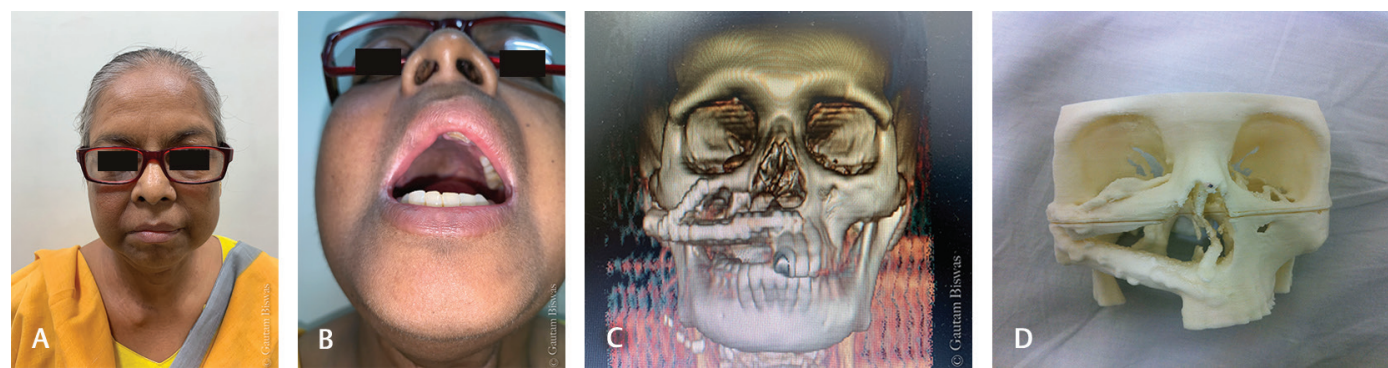

Fig. 5 (A-D) Post maxillectomy reconstruction for a type II (Cordeiro) defect reconstructed using a fibular strut for the horizontal buttress along with 3D reconstructed CT image and 3D resin model showing the disposition of the fibula in a "V" pattern reconstruction. 3D, three-dimensional.
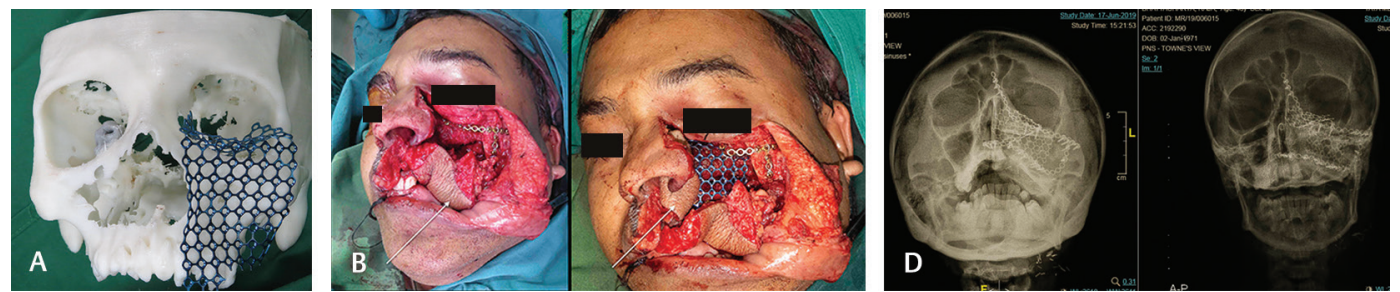

Fig. 6 (A) Precontouring of titanium mesh over a 3D printed model. (B) Intraoperative placement over a chimeric free osteocutaneous fibula flap. (C) Postoperative X-ray. 3D, three-dimensional.

gets retracted within them, affecting aesthetics. Titanium mesh, either precontoured on a 3D printed model or an intraoperatively contoured mesh are some choices ( - Fig. 6A-C).

Difficulties using the fibula in Cordeiro type III or Brown Class 3 defects have been highlighted by various authors. While Cordeiro suggests reconstruction using soft tissue flaps Brown proposed the use of the DCIA contoured to form the orbital rim and bony support to the cheek. Various combinations using nonvascularized grafts and titanium mesh have been described.

\section{Reconstructing the Orbital Floor}

The orbital floor and medial wall formed by the roof of the Maxilla provide a shelf supporting the orbital contents. The position of this floor is critical as this would decide whether the contents are in correct alignment, positioned inferiorly would result in enophthalmos and possibly diplopia, whereas upwardly displaced, the globe displaces up with the increased scleral show.

Orbital floor reconstruction options range from using a vascular bone strut to mimic the rim, and use of titanium mesh, bioabsorbable implants, calvarial bone, split rib, or iliac crest grafts to form the floor ${ }^{15,16}$ ( $\sim$ Fig. 7 ).

Connolly et al reported the use of osteocutaneous RAFF for maxillectomy defects, wherein they reconstructed the orbital rim in $82 \%$ of the patients augmenting this with a titanium mesh and absorbable plate in $42 \%$ of this group. They reported a comparable result using a calvarial bone graft or iliac crest with rectus abdominis muscle. These resections showed a higher rate of diplopia and lower eyelid ectropion, affecting their quality of life. ${ }^{15}$ Cordeiro and Santamaria ${ }^{8}$ reported 14 patients who underwent resection of the orbital floor, with

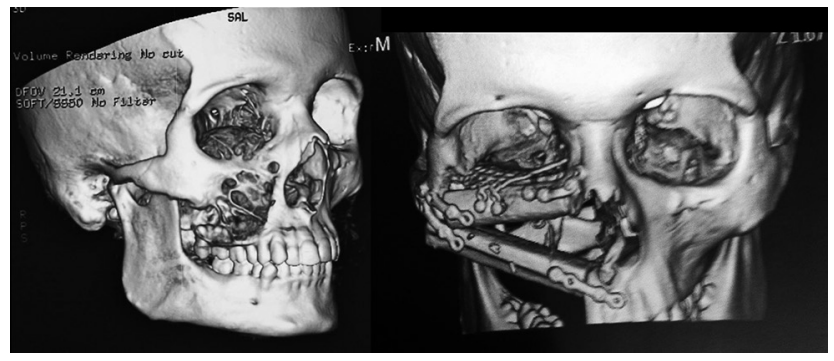

Fig. 7 CT scan-giant cell tumor maxilla. Post reconstruction with a fibula with a precontoured titanium mesh for the orbital floor.

one patient developing vertical dystopia and another diplopia. Ten of the 14 patients developed lower eyelid ectropion. ${ }^{16}$

Options for recreating the orbital floor include using a vascularized segment of the fibula in tandem with the alveolar reconstruct for the rim and fixing a contoured titanium plate for recreating the floor. The presence of orbital contents and soft tissue renders the intraoperative contouring of the orbital plate difficult. Ideally, a preoperative 3D printed model provides a template by which the implant may be precontoured ( - Fig. 6A).

A precontoured titanium mesh ( $\mathbf{- F i g . 6 B}$ ) may also be used to reconstruct the maxillary body and fixed to the alveolar construct of the vascularized fibula. This can extend into the orbit, recreating the floor and medial wall. A drawback of using the mesh externally is the tethering of the skin, especially, following radiotherapy. When using this technique, the authors interpose a part of the deepithelialized skin paddle over the implant, thus avoiding this problem and also augmenting the soft tissue ( - Fig. 8). 


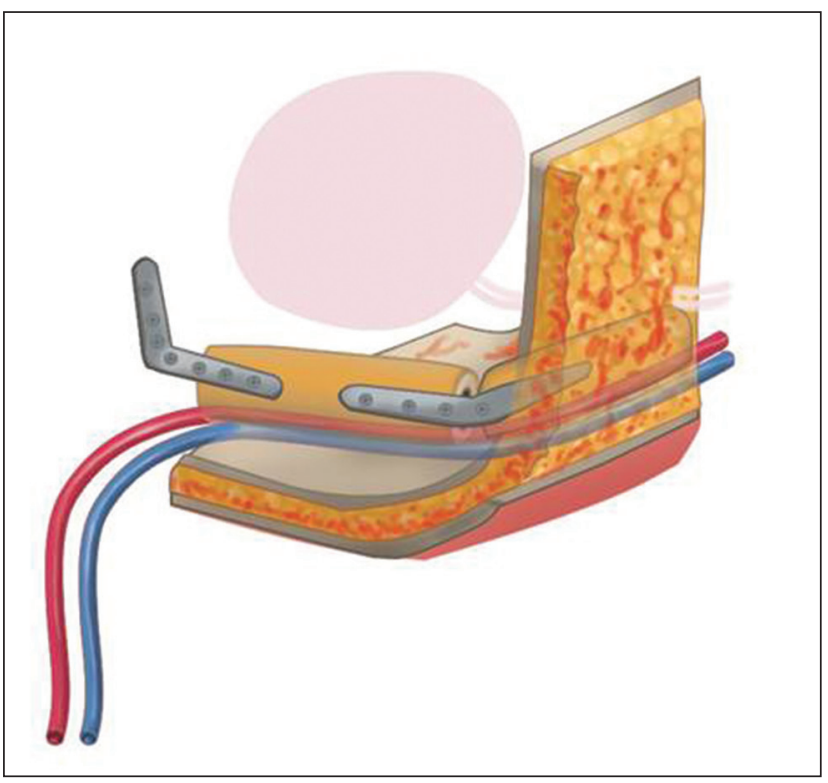

Fig. 8 Diagram illustrating augmenting the anterior surface of a titanium mesh reconstruction using a de-epithelized extension of the skin component of the free fibula flap.

Soft tissue free flaps for type IV defects have been suggested by Cordeiro and Santamaria. As the orbital content involved by the tumor are removed, malpositioning of the globe or ectropion is no more a concern. Commonly the rectus abdominis or anterolateral thigh flap with vastus lateralis are used to fill up this defect. ${ }^{8}$

The long-term consequences of using only a soft tissue reconstruction are that with atrophy of the muscle and post-radiotherapy changes, coupled with effects of gravity the remnant soft tissue sags and a significant contour deformity occurs in the cheek.

Combining the bone reconstruction restores midfacial height, facial width, and projection, as well as adequate bone stock for osseointegration. ${ }^{6}$ Brown suggested the use of the DCIA flap, which does satisfy this problem by providing both bone and soft tissue. The shortcomings of the DCIA is its short pedicle, often requiring a graft. ${ }^{9}$

\section{Recipient Vessels}

Another challenge in midfacial reconstruction, often influencing the choice of the flap, is the selection of the recipient vessels. A commonly favored option is the use of facial vessels. ${ }^{17}$

In fibular osteocutaneous flaps where the orbital rim is reconstructed, multiple osteotomies, including removing segments of bone to allow folding, uses up the available pedicle length. An unpublished study by the author showed that an average length of $22 \mathrm{~cm}$ of the fibula is available, of which the available pedicle finally was 8 to $9 \mathrm{~cm}$. Dalgorf et al noted that a length of 10 to $12 \mathrm{~cm}$ of the pedicle is necessary to reach the recipient vessels in the neck. ${ }^{17}$ The pedicle just reaches the mandibular border, where the facial vessels are of significantly smaller diameter as compared with the proximal peroneal pedicle. This discrepancy needs addressing. The vessels are also at different planes adding to this difficulty.

When soft tissue flaps are used, the choices are the rectus abdominis, anterolateral thigh, or latissimus dorsi flaps, as they have long pedicles which can safely reach the neck.

It is imperative to check the reach of the pedicle as it may require extending, by intramuscular dissection, as is sometimes required, especially when, using the contralateral neck. ${ }^{8}$

The superficial temporal vessels are also an alternative. Tortuosity, increased spasm, smaller diameter, and a single thin-walled vein have been cited as disadvantages. ${ }^{17}$ The DCIA flap has a pedicle length of around $4 \mathrm{~cm}$, ${ }^{6}$ hence anastomosis needs to be done over the mandibular bone ${ }^{9}$ or vein grafts used for both artery and vein. ${ }^{9}$ Secondary reconstructions, recurrent disease, previously operated and radiated neckthe vessel depleted neck pose challenges in finding suitable vessels. Use of the transverse cervical vessels, contralateral neck vessels, and the acromion-thoracic pedicle has all been anecdotally reported. ${ }^{1}$

\section{Central Midface Reconstruction}

The central midface extends from the root of the nose down to the alveolus inferiorly and includes the upper lip. For ease of understanding, it can be seen to be triangular in shape with its apex separated from the anterior skull base by the nasoethmoidal complex, and the base formed by the upper lip and central maxilla. The central midface is mostly represented by the nose supported on the skeletal foundation of the maxilla, bordering the piriform aperture, and the intrinsic osteocartilaginous support.

Tumors in this area often extend into the anterior skull base, making planning and reconstruction much more complex. Tumors in this area can involve several structures and may extend into the lateral midface as well as the medial orbits and skull base. It is an advantage to visualize this involvement using 3D images and utilize 3D printed models to plan the reconstructive options as well as its stages if required.

Cordeiro and Santamaria suggested that these central defects are best addressed in stages while Horace, in his published experience of 24 years of 54 patients, suggested that the reconstruction should be primarily performed and he illustrated two patients where a sequential-linked flow-through flap was performed to reconstruct both the maxilla and nose in the same sitting. ${ }^{8,11}$

Conventional multistaged reconstruction in this area ${ }^{24-26}$ using local flaps combined with rib cartilage grafts is often not an option following malignant tumor extirpations. The stages need to be compressed within the stipulated 6 weeks between resection and radiation. The alternative is to delay the reconstruction well after the completion of the primary management of the disease, which often includes surgery, radiotherapy, and occasionally chemotherapy and may extend to many months. In the intervening period, an external prosthesis may be used (-Fig. 1). 
Burget and Walton outlined the principles in the planning and design of free flaps in nasal reconstruction. All their reconstructions were done secondarily. Multipleislanded radial forearm flaps with cartilage support and midline forehead flaps were commonly used in stages. An average of seven carefully planned procedures over a mean period of over 26 months was required to complete the reconstruction..$^{25}$

These are often not practical in resource-constrained environments, apart from the stigma associated with such deformities.

Full-thickness defects of the central midface, categorized as "complex 3D defects" by O"zkan et al ${ }^{27}$ necessitate a composite reconstruct of lining, support, and cover. Lining losses are usually extensive, often extending into the maxilla. The bony platform first needs to be re-established, on which the nasal structure would need to rest. Large lining losses can be replaced with a free radial forearm flap, using a folded extension to provide cover in the first stage. In the second stage, a forehead flap combined with rib cartilage graft support is performed. It is ideal if all procedures are completed and the wound healed prior to radiotherapy.

These defects also encompass the columella, reconstruction of which, needs to be planned. Primarily reconstructing the columella with the RAFF or combining this with a forehead flap may create some difficulties. The authors tend to confine the initial transfer to securing a safe transfer of the flap possibly with some redundant soft tissue and then replacing that which is redundant, using a part of it to create the posterior columella and then using a forehead flap overlying this sandwiching the osteocartilagenous rib construct to form the nose ( - Fig. 9A-G).

Tunneling and positioning of the pedicle, disposition of the soft tissue, using any residual nasal tissue, all need to be factored in while planning. The aim is not only to reconstruct an aesthetically pleasing nose but also to provide a functionally adequate nasal airway. The forehead flap needs to be adequately planned so as to drape the framework without tension.

\section{Imaging and 3D Planning}

Visualizing the anatomical extent of the lesion and the structures which would require reconstruction is critical for planning. Imaging modalities such as CT and MRI are generally routine investigative tools in craniofacial lesions, necessary for diagnosis, staging, and assessing resectability. ${ }^{28}$ They also identify the need for involving other specialties like neurosurgery, and in understanding the outcomes. ${ }^{29}$ Both these two imaging modalities complement each other, each having their own nuances. The CT is more precise in skeletal visualization, with the MRI delineating the soft tissue structures better.

Software aided conversion of high-resolution CT data to 3D CT imaging allows a clearer understanding when viewed in three dimensions, allowing rotation of the image and visualization from different angles ( - Fig. 10A-D). Presurgically planning the cuts, precise measurement of the size, and fabricating cutting guides are some of its applications. ${ }^{30,31}$ Virtual surgical planning using CT images and 3D printed models is now increasingly being integrated into these surgeries to assist the surgical team, plan, and perform a mock surgery, and prebend plates. These precontoured plates allow quick fixation while the flap is still attached to the donor site. This facilitates more cohesive teamwork, reduces ischemia time, and shortens the overall operating time. ${ }^{22,30,32}$

3D models are both educative to the patient as well as the surgical team. Preprinting implants are also being explored with the future being directed toward printing patient-specific implants which can be biologically integrated.
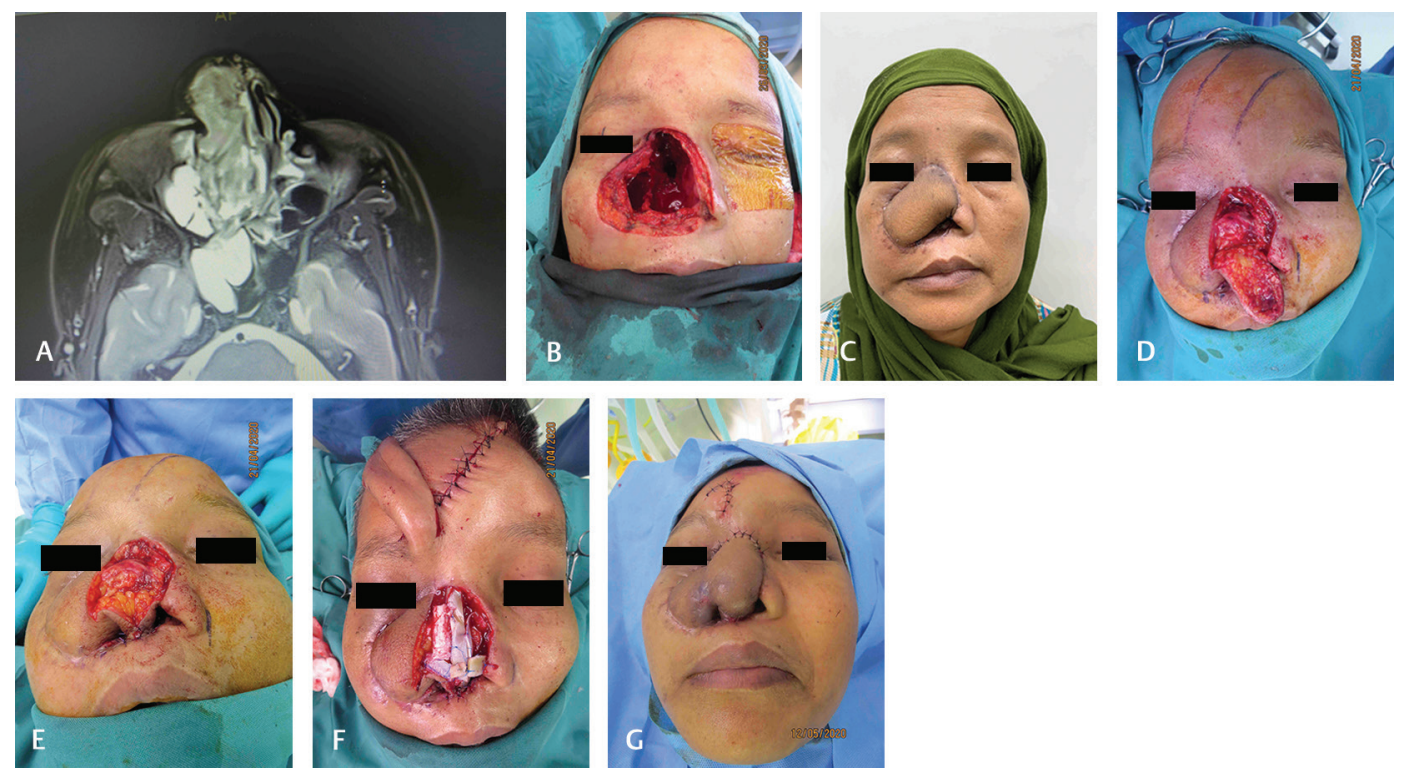

Fig. 9 (A-C) Nasomaxillary tumor excision and primary reconstruction with a sandwich free radial forearm flap, to address lining and cover. (D-F) Second-stage nasal reconstruction, hinging the outer portion of RAFF, to reconstruct the posterior columella, placement of cartilaginous framework, and draping with a midline forehead flap. (G) Postoperative result. RAFF, rectus abdominis free flap. 

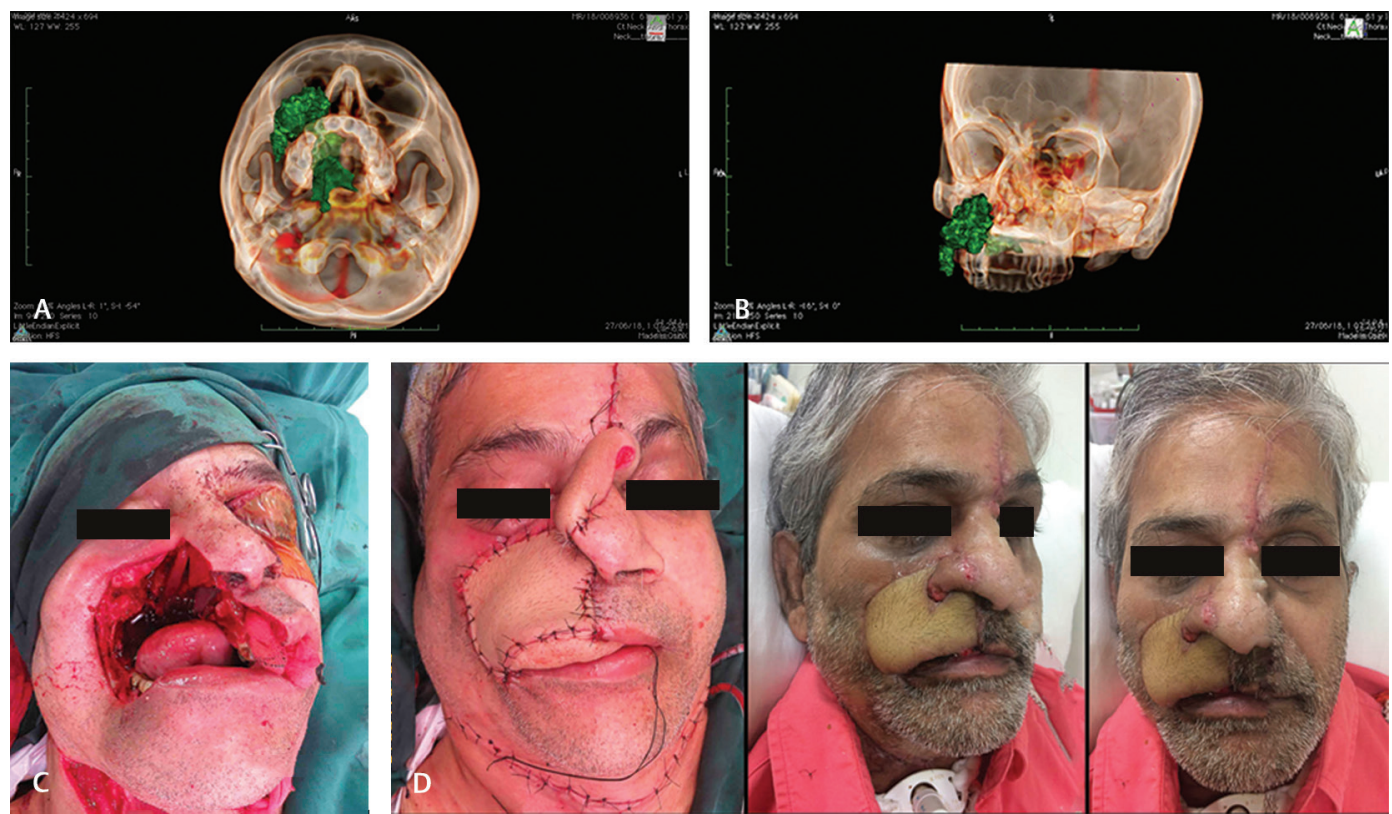

Fig. 10 (A,B) 3D imaging to visualize the anatomical involvement. (C) Post excision defect reconstructed using fibular osteocutaneous flap for alveolus and palate, with a contoured titanium mesh for the anterolateral maxilla. (D) Alae reconstructed by a staged midline forehead flap and the lip using a free RAFF. 3D, three-dimensional; RAFF, rectus abdominis free flap.

Our practice is to use 3D printed models to precontour titanium mesh for the anterolateral maxilla and orbital floor and to precontour low profile reconstruction plates ( - Fig. 11A-D).

The applications of 3D printing technology have been summarized thus ${ }^{31}$ :

Contour models: which are the patient-specific exact replica of the skeletal system used to precontour plates. This has an advantage over intraoperative bending where soft tissues, bleeding, anatomical distortions because of trauma or tumor interfere with the process.

Guides: which are the patient-specific templates to mark areas to be drilled or cut.

Splints: predesigned to allow exact relocation, especially to provide occlusal alignment.

Implants: which are the patient-specific constructs implanted into the patient or the creation of casts in the design of finally-constructed implantable and biologically integrated implants. ${ }^{33}$

These techniques are especially useful in secondary reconstructions, where the tissues are distorted and displaced. They can be virtually restored to their normal position and a template created of the true defect "mirroring" the normal side ( - Fig. 12A-F). ${ }^{12,22,34,}$

A few studies have compared 3D printing versus conventional techniques, with some showing an advantage while others were showing an equivalent result. ${ }^{31}$

\section{Secondary Reconstruction}

Further surgical procedures ${ }^{21,22}$ may be necessary to correct the shortcomings of the primary procedure, the changes following radiation and to achieve functional or aesthetic improvement. Plate exposure, contour defects, orbital
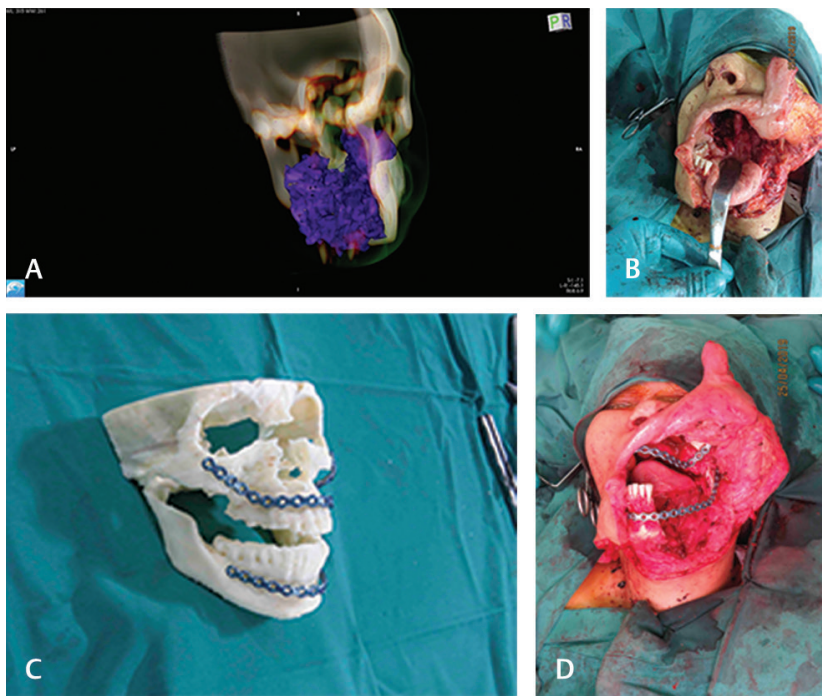

Fig. 11 (A-D) Nasopharyngeal malignancy requiring a bilateral maxillectomy and right segmental mandibulectomy. Planning done using 3D imaging and printing. Prebending of titanium reconstruction plates. Reconstruction using bilateral fibula osteocutaneous flaps, combined with titanium mesh for the maxilla. 3D, three-dimensional.

deficits, and nasal deficits are some common problems. Achieving all the reconstructive goals in a single stage is often not feasible and secondary procedures are often necessary. ${ }^{25,35}$

In these situations, traditionally the reconstructive requirements were planned using normal bony landmarks. These often are altered, hence virtual planning is an option. John Pang et al described an interesting concept of staged maxillary reconstruction, where in the first stage, the extirpation surgery is done without neck dissection. A "delayed immediate reconstruction" using a CAD cam designed free 
fibula osteocutaneous flap was then done combined with a neck dissection. The advantage claimed is that of the staged reconstruction having an assured negative margin. The delay in neck dissection does not affect survival and allows anastomosis in a previously unoperated neck. The interval between the two stages allows fabricating the 3D printed model and its guides. ${ }^{36}$

\section{Dental Implants}

The final summation is to achieve dental restoration, which is not only functionally restorative but also has aesthetic implications. The introduction of osseointegrated implants has opened up another avenue for improvement as these can now hold dental implants or prostheses. The reconstructed fibula, ilium or scapula provide an adequate bony stock for implants. ${ }^{37}$ If adequate normal dentition or alveolus remains on the healthy side, it may be used to carry a prosthetic-based denture ( - Fig. 13A,B).

Foster et al published their experience in reconstructing 26 patients with complex midfacial defects where implants were used in 14 of 18 patients who had undergone alveolar ridge reconstruction. Of these, eight received dentures and six osseointegrated implants, three being at the time of initial surgery. The authors suggested removable prosthesis if more than half the palate was preserved and a permanent denture bearing prosthesis where the palatal defect exceeded more than half the palate. ${ }^{28}$
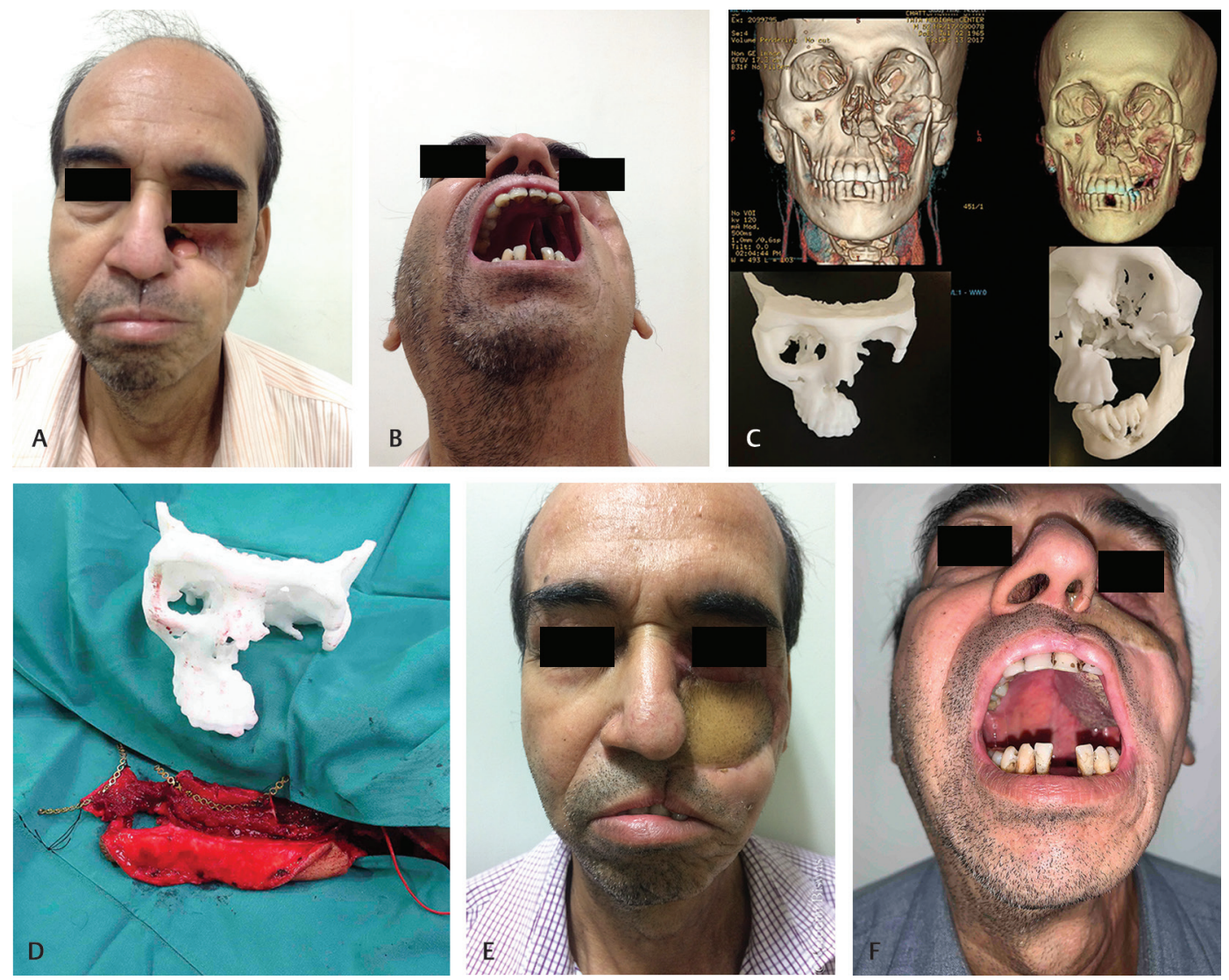

Fig.12 (A-F) Secondary defect following disruption of skin incision for an unreconstructed maxillectomy. Planning using a 3D CT image and $3 \mathrm{D}$ resin model, for measurements and prebending plates. 3D, three-dimensional.
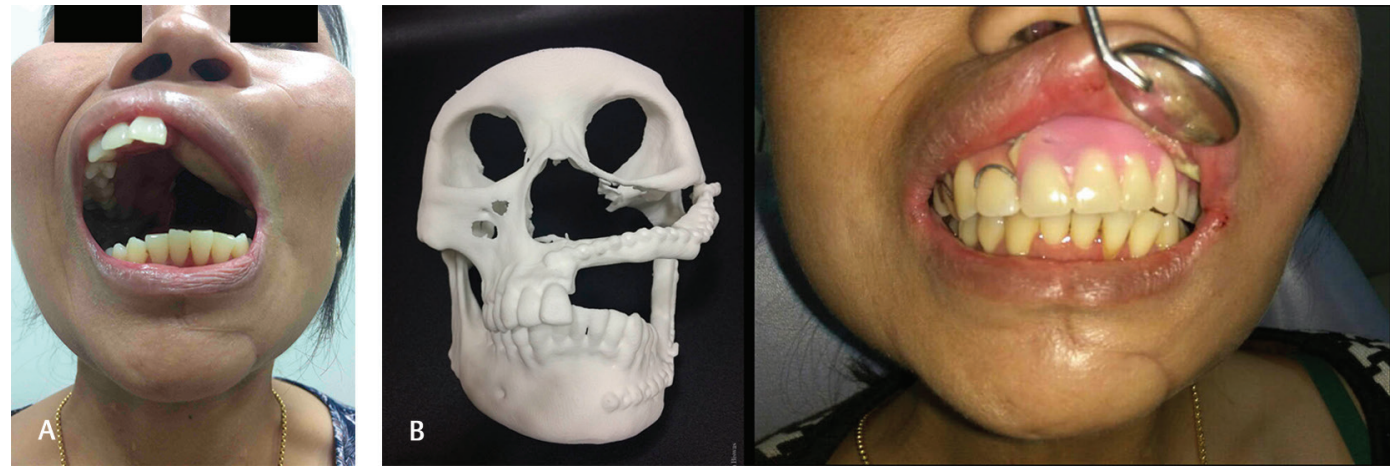

Fig. 13 (A) Post reconstruction for a combined maxillary mandibular defect reconstructed using a single fibula. (B) 3D printed model post surgery. The result following placement of an implant-based prosthesis. 3D, three-dimensional. 
In general, osseointegrated implants are placed approximately 4 to 6 months after the procedure, being delayed even further if the patient undergoes irradiation. ${ }^{38}$ Radiation between 60 and $70 \mathrm{~Gy}$ was considered a relative contraindication for the placement of implants. In post irradiated cases, it is generally agreed that implants can be placed 6 to 12 months following radiotherapy. ${ }^{38}$

Primary implants can be placed in the harvested free fibula using computer guidance at the time of harvest or after inset. ${ }^{39}$ The success rate for implants placed on irradiated areas is reported to be $91.3 \%$ between 1 and 5 years. ${ }^{40}$

The "jaw in a day" concept proposed by Patel et al in 2012 was to provide immediate placement of implants and combining with a provisional fixed dental prosthesis in the same sitting. ${ }^{41}$

\section{Conclusion}

Microsurgical procedures, combining multidisciplinary teams, incorporating virtual surgical planning open up new avenues in achieving reconstructive goals. There are still areas of controversy and debate and unanswered questions in midfacial reconstruction.

\section{Conflict of Interest}

None declared.

\section{Acknowledgments}

The author is thankful toward all his colleagues and patients who have helped him understand the complexities.

\section{References}

1 Neligan PC, Lipa JE, Reconstruction of the midface. In: Evans GRD, Butler CE, eds. Procedures in Reconstructive Surgery. Philadelphia: Saunders; 2009: 139-152

2 Taylor GI, Corlett RJ, Ashton MW. The evolution of free vascularized bone transfer: a 40-year experience. Plast Reconstr Surg 2016;137(4):1292-1305

3 Watanabe K, Takahashi N, Morihisa Y, et al. Maxillary reconstruction using chimeric flaps of the subscapular artery system without vein grafts and the novel usage of chimeric flaps. J Reconstr Microsurg 2013;29(9):601-606

4 Peng X, Mao C, Yu GY, Guo CB, Huang MX, Zhang Y. Maxillary reconstruction with the free fibula flap. Plast Reconstr Surg 2005;115(6):1562-1569

5 Iyer S, Thankappan K. Maxillary reconstruction: current concepts and controversies. Indian J Plast Surg 2014;47(1): 8-19

6 Andrades P, Militsakh O, Hanasono MM, Rieger J, Rosenthal EL. Current strategies in reconstruction of maxillectomy defects. Arch Otolaryngol Head Neck Surg 2011;137(8):806-812

7 Okay DJ, Genden E, Buchbinder D, Urken M. Prosthodontic guidelines for surgical reconstruction of the maxilla: a classification system of defects. J Prosthet Dent 2001;86(4):352-363

8 Cordeiro PG, Santamaria E. A classification system and algorithm for reconstruction of maxillectomy and midfacial defects. Plast Reconstr Surg 2000;105(7):2331-2346, discussion 2347-2348

9 Brown JS. Maxillary reconstruction. Indian J Plast Surg 2007; 40:35-43

10 Chang DW, Langstein HN. Use of the free fibula flap for restoration of orbital support and midfacial projection following maxillectomy. J Reconstr Microsurg 2003;19(3):147-152
11 Costa $\mathrm{H}$, Zenha $\mathrm{H}$, Sequeira $\mathrm{H}$, et al. Microsurgical reconstruction of the maxilla: algorithm and concepts. J Plast Reconstr Aesthet Surg 2015;68(5):e89-e104

12 Alam D, Ali Y, Klem C, Coventry D. The evolution of complex microsurgical midface reconstruction: a classification scheme and reconstructive algorithm. Facial Plast Surg Clin North Am 2016;24(4):593-603

13 Eckardt A, TeltzrowT, Schulze A, Hoppe M, Kuettner C. Nasalance in patients with maxillary defects-reconstruction versus obturation. J Craniomaxillofac Surg 2007;35(4-5):241-245

14 Rieger J, Bohle Iii G, Huryn J, Tang JL, Harris J, Seikaly H. Surgical reconstruction versus prosthetic obturation of extensive soft palate defects: a comparison of speech outcomes. Int J Prosthodont 2009;22(6):566-572

15 Connolly TM, Sweeny L, Greene B, Morlandt A, Carroll WR, Rosenthal EL. Reconstruction of midface defects with the osteocutaneous radial forearm flap: evaluation of long term outcomes including patient reported quality of life. Microsurgery 2017;37(7):752-762

16 Santamaria E, Cordeiro PG. Reconstruction of maxillectomy and midfacial defects with free tissue transfer. J Surg Oncol 2006;94(6):522-531

17 Dalgorf D, Higgins K. Reconstruction of the midface and maxilla. Curr Opin Otolaryngol Head Neck Surg 2008;16(4): 303-311

18 Bidros RS, Metzinger SE, Guerra AB. The thoracodorsal artery perforator-scapular osteocutaneous (TDAP-SOC) flap for reconstruction of palatal and maxillary defects. Ann Plast Surg 2005;54(1):59-65

19 Brown JS. Deep circumflex iliac artery free flap with internal oblique muscle as a new method of immediate reconstruction of maxillectomy defect. Head Neck 1996;18(5):412-421

20 Futran ND, Wadsworth JT, Villaret D, Farwell DG. Midface reconstruction with the fibula free flap. Arch Otolaryngol Head Neck Surg 2002;128(2):161-166

21 Fisher M, Dorafshar A, Bojovic B, Manson PN, Rodriguez ED. The evolution of critical concepts in aesthetic craniofacial microsurgical reconstruction. Plast Reconstr Surg 2012; 130(2):389-398

22 Jang WH, Lee JM, Jang S, Kim HD, Ahn KM, Lee JH. Mirror image based three-dimensional virtual surgical planning and three-dimensional printing guide system for the reconstruction of wide maxilla defect using the deep circumflex iliac artery free flap. J Craniofac Surg 2019;30(6):1829-1832

23 Lenox ND, Kim DD. Maxillary reconstruction. Oral Maxillofac Surg Clin North Am 2013;25(2):215-222

24 Parrett BM, Pribaz JJ. An algorithm for treatment of nasal defects. Clin Plast Surg 2009;36(3):407-420

25 Burget GC, Walton RL. Optimal use of microvascular free flaps, cartilage grafts, and a paramedian forehead flap for aesthetic reconstruction of the nose and adjacent facial units. Plast Reconstr Surg 2007;120(5):1171-1207, discussion 1208-1216

26 Pribaz JJ, Weiss DD, Mulliken JB, Eriksson E. Prelaminated free flap reconstruction of complex central facial defects. Plast Reconstr Surg 1999;104(2):357-365, discussion 366-367

27 O"zkan O, Coşkunfirat OK, O"zkan O. Midface reconstruction. Semin Plast Surg 2010;24(2):181-187

28 Foster RD, Anthony JP, Singer MI, Kaplan MJ, Pogrel MA, Mathes SJ. Reconstruction of complex midfacial defects. Plast Reconstr Surg 1997;99(6):1555-1565

29 Fanucci E, Fiaschetti V, Fusco N, et al. Role of diagnostic imaging to malignant sinus tumors in presurgical staging. Oral Implantol (Rome) 2008;1(2):56-65

30 Saad A, Winters R, Wise MW. Dupin CL, St Hilaire H. Virtual surgical planning in complex composite maxillofacial reconstruction. Plast Reconstr Surg 2013;132(3):626-633

31 Jacobs CA, Lin AY. A new classification of three-dimensional printing technologies: systematic review of three-dimensional 
printing for patient-specific craniomaxillofacial surgery. Plast Reconstr Surg 2017;139(5):1211-1220

32 He Y, Zhu HG, Zhang ZY, He J, Sader R. Three-dimensional model simulation and reconstruction of composite total maxillectomy defects with fibula osteomyocutaneous flap flow-through from radial forearm flap. Oral Surg Oral Med Oral Pathol Oral Radiol Endod 2009;108(6):e6-e12

33 Mertens C, Löwenheim H, Hoffmann J. Image data based reconstruction of the midface using a patient-specific implant in combination with a vascularized osteomyocutaneous scapular flap. J Craniomaxillofac Surg 2013;41(3):219-225

34 Stranix JT, Stern CS, Rensberger M, et al. A virtual surgical planning algorithm for delayed maxillomandibular reconstruction. Plast Reconstr Surg 2019;143(4):1197-1206

35 Rodriguez ED, Bluebond-Langner R, Park JE, Manson PN. Preservation of contour in periorbital and midfacial craniofacial microsurgery: reconstruction of the soft-tissue elements and skeletal buttresses. Plast Reconstr Surg 2008; 121(5):1738-1747, discussion 1748-1749

36 Pang JH, Brooke S, Kubik MW, et al. Staged reconstruction (delayed-immediate) of the maxillectomy defect using CAD/ CAM technology J Reconstruct Microsurg 2018;34(3):193-199
37 Yilmaz M, Vayvada H, Menderes A, Demirdover C, Kizilkaya A. A comparison of vascularized fibular flap and iliac crest flap for mandibular reconstruction. J Craniofac Surg 2008; 19(1):227-234

38 Smith Nobrega A, Santiago JF Jr, de Faria Almeida DA, Dos Santos DM, Pellizzer EP, Goiato MC. Irradiated patients and survival rate of dental implants: a systematic review and meta-analysis. J Prosthet Dent 2016;116(6):858-866

39 Chang YM, Santamaria E, Wei FC, et al. Primary insertion of osseointegrated dental implants into fibula osteoseptocutaneous free flap for mandible reconstruction. Plast Reconstr Surg 1998;102(3):680-688

40 Patel SY, Kim DD, Ghali GE. Maxillofacial reconstruction using vascularized fibula free flaps and endosseous implants. Oral Maxillofac Surg Clin North Am 2019;31(2):259-284

41 Patel A, Levine J, Brecht L, Saadeh P, Hirsch DL. Digital technologies in mandibular pathology and reconstruction. Atlas Oral Maxillofac Surg Clin North Am 2012;20(1):95-106 